\title{
Troubles dans les apprentissages : neurosciences cognitives et difficultés scolaires
}

Disorders in learning : cognitive neurosciences and academic difficulties

Stanislas Morel

\section{(2) OpenEdition}

1 Journals

Édition électronique

URL : http://journals.openedition.org/ress/3526

DOI : $10.4000 /$ ress.3526

ISBN : $1663-4446$

ISSN : $1663-4446$

Éditeur

Librairie Droz

Édition imprimée

Date de publication : 15 mai 2016

Pagination : 221-247

ISSN : 0048-8046

\section{Référence électronique}

Stanislas Morel, «Troubles dans les apprentissages : neurosciences cognitives et difficultés scolaires », Revue européenne des sciences sociales [En ligne], 54-1 | 2016, mis en ligne le 15 mai 2019, consulté le 03 janvier 2020. URL : http://journals.openedition.org/ress/3526 ; DOI : 10.4000/ress.3526 


\title{
TROUBLES DANS LES APPRENTISSAGES: NEUROSCIENCES COGNITIVES ET DIFFICULTÉS SCOLAIRES
}

\author{
STANISLAS MOREL \\ Université de Saint-Étienne \\ stanislas.morel@univ-st-etienne.fr
}

\begin{abstract}
Résumé. Cet article porte sur le recours croissant aux neurosciences cognitives pour interpréter et traiter les difficultés scolaires. Ces usages s'expliquent d'abord par le travail de construction des neurosciences cognitives en discipline pragmatique orientée vers l'action à travers un matérialisme scientifique présenté comme ouvert et libéré des a priori idéologiques. Ils ont été ensuite favorisés par la constitution des «troubles spécifiques des apprentissages» en problème de santé publique dont l'étude et la résolution supposent l'action conjointe des chercheurs et des professionnels de santé. L'entreprise de légitimation de la réponse des neurosciences aux difficultés d'apprentissage a néanmoins bénéficié d'autres relais. Deux d'entre eux seront ici examinés, à savoir, d'une part, les hommes politiques, hauts fonctionnaires et experts chargés d'élaborer et de mettre en œuvre une réforme pédagogique, et, d'autre part, les parents d'élèves en difficulté dans le cadre scolaire.
\end{abstract}

Mots-clés: associations de parents, difficultés d'apprentissage, échec scolaire, politiques éducatives, psychologie cognitive, neurosciences, sociologie des sciences.

\begin{abstract}
This article deals with the increasing uses of cognitive neurosciences to interpret and address learning difficulties. These uses are first explained by the progressive recognition of cognitive neurosciences as an action-oriented pragmatic discipline, whose scientific materialism is supposed to be free from ideological a priori. They were also fostered by the construction of "specific learning disorders" as a public health problem whose study and resolution suppose the joint action of researchers and health professionals. The legitimation of the cognitive neurosciences approach of school difficulties nevertheless was supported by other actors. Two sets of actors will be examined here, namely, on the one hand, politicians, top civil servants and experts charged to work out and implement education policies, and, on the other hand, the parents of students with learning difficulties.
\end{abstract}

Keywords: cognitive psychology, learning difficulties, parent association, neurosciences, school failure, sociology of science. 
On assiste en France à un renouveau des interprétations faisant des difficultés scolaires la conséquence de dysfonctionnements cognitifs, voire de «troubles spécifiques des apprentissages » d'origine neurologique. Ce renouveau est directement lié au déclin de certaines interprétations sociologisantes ou psychologisantes $^{1}$ relativement dominantes dans la période précédente et au développement des neurosciences cognitives (NC, ci-après) depuis les années 1980 (Chamak et Moutaud, 20I4; Lemerle, 20I4a; Rose et Abi-Rached, 20I3).

Néanmoins, tant la diffusion des NC hors des laboratoires où elles sont produites que leur appropriation par différents groupes sociaux ou leur application à des domaines variés (éducation, économie, psychiatrie, justice, esthétique, etc.), sont loin d'aller de soi. Cherchant à mieux comprendre ce phénomène de diffusion, d'appropriation et d’application, cet article prend pour objet les usages sociaux des NC dans le domaine particulier des apprentissages scolaires et, plus particulièrement, des difficultés qui leur sont associées. Il propose d’articuler une analyse interne (ce que ces usages doivent aux spécificités de l’approche scientifique) et une analyse externe (ce qu'ils doivent au problème auquel les recherches sont appliquées et aux groupes sociaux qui y recourent).

Deux caractéristiques de la construction scientifique des NC conditionnent largement ses usages potentiels dans le domaine des apprentissages scolaires. La première est que les NC sont résolument orientées vers l'action à travers un matérialisme scientifique présenté comme libéré des a priori idéologiques et politiques. Contrairement à certaines idées reçues, elles tendent, au moins aujourd'hui, à reléguer au second plan les questionnements ontologiques (quelle est la cause première des difficultés d’apprentissage ?) et à privilégier, d'une part, une approche descriptive des processus cognitifs engagés dans les apprentissages - et de leurs «corrélats anatomiques » - et, d'autre part, l'élaboration d'«interventions », qui ont pour objectif de maximiser les performances cognitives des individus et dont l'efficacité est expérimentalement démontrée. Ainsi, outre la fascination associée à la découverte des mystères du cerveau, une partie de l'attrait actuellement exercé par les NC est étroitement liée à l'espoir, 
entretenu à la fois par les chercheurs et par leurs auditoires, de résolution totale ou partielle de certains «problèmes » qui perturbent le fonctionnement de nos sociétés. La seconde caractéristique tient à la capacité des NC à étudier à la fois le «normal» et le «pathologique», voire à brouiller les frontières entre ces deux «registres». Ce large empan de la recherche est à l'origine d'une multiplication des applications possibles et permet aux chercheurs NC de disposer de plusieurs leviers pour diffuser leurs recommandations. Dans le domaine de l'apprentissage de la lecture par exemple, la mise en évidence des processus cognitifs et des «neurones de la lecture» (Dehaene, 2007) conduit à des préconisations dans le domaine de la pédagogie générale (Dehaene, 20I I), tandis que la découverte de pathologies largement répandues comme la dyslexie débouche sur la construction de problèmes de santé publique. Ces deux types de problématisations des difficultés d’apprentissage actionnent des leviers et mobilisent des acteurs différents et complémentaires.

Néanmoins, les potentialités d’appropriation et d’application engendrées par cette construction scientifique ne se concrétisent qu'à certaines conditions, étudiées dans une seconde partie de l'article. Comprendre le recours massif aux analyses des difficultés d'apprentissage produites par les NC suppose notamment de prendre en considération leur adéquation à la formulation actuellement dominante du problème de l'échec scolaire et aux principales réponses qui lui sont apportées par les politiques publiques. L'étude de la diffusion des NC dans la communauté éducative doit aussi analyser le rôle de certains acteurs comme les familles - à commencer par les parents d'élèves en difficulté - qui, à l'encontre d'une vision réduisant la médicalisation à un moyen de contrôle social des populations (Foucault, 1988; Donzelot, 1977), jouent aujourd'hui, au moins pour certaines d'entre elles, un rôle actif dans la diffusion des interprétations médico-psychologiques de l'échec scolaire les plus compatibles avec leurs propres représentations des difficultés de leur enfants et avec leurs intérêts (Garcia, 20I3 ; Lignier, 20I2; Morel, 20I4). 


\section{I.EN QUOI LES CARACTÉRISTIQUES D'UNE APPROCHE SCIENTIFIQUE CONDITIONNENT SES USAGES SOCIAUX?}

Les NC peuvent être définies comme l'«ensemble des disciplines qui ont pour objet d'établir la nature des relations entre la cognition et le cerveau » (Tiberghien, 2002). Résultant de la convergence de chercheurs en psychologie cognitive et en neurosciences (Plas, 20II), elles regroupent un ensemble hétérogène de chercheurs et de praticiens : biologistes, neurologues, pédiatres, pédopsychiatres, neuropsychologues, psychologues cognitivistes, linguistes, spécialistes de l'intelligence artificielle, de l'imagerie cérébrale ou de la modélisation, etc. Initialement dominées par la psychologie cognitive, elles s'organisent de plus en plus, depuis une vingtaine d’années, autour des neurosciences, qui, avec les progrès des techniques d’imagerie cérébrale, ont pris une place centrale (Proust, 1992). Ce regroupement s'est progressivement institutionnalisé et on compte désormais de très nombreux laboratoires ou revues scientifiques qui se revendiquent des NC, en particulier aux États-Unis.

Parmi tous les champs de recherche des NC, l'étude du développement de la cognition chez l'enfant est sans doute un des plus explorés. Comment l'enfant apprend-il à parler, à lire, à écrire ou à compter? Quels raisonnements met-il en œuvre? Comment mémorise-t-ils les informations qui lui sont transmises ? Comment apprend-il à être attentif, à focaliser son attention sur certaines tâches? Autant de questions qui ont fait l'objet de très nombreux travaux et qui constituent un des points nodaux des recherches en NC. Les enfants sont des sujets d'étude d'autant plus intéressants que la plasticité de leur cerveau en construction se prête particulièrement à la démarche expérimentale des chercheurs en NC. Ces derniers peuvent observer le développement des processus cognitifs et de leurs bases neurales en fonction des tâches proposées aux enfants. L'objectif de cet article étant d’analyser les usages sociaux des NC, nous n’entrerons pas dans une présentation détaillée de leurs théories de l’apprentissage. Il s'agira plutôt de mettre en évidence ce qui, dans ces théories, est susceptible de susciter l'adhésion et de favoriser leur appropriation en dehors de l'univers scientifique où elles sont produites. 


\section{I.I.DES NEUROSCIENCES COGNITIVES ORIENTÉES VERS L'ACTION}

Les chercheurs en NC qui travaillent actuellement sur la question des apprentissages sont, qu'ils le veuillent ou non, pris dans les controverses qui ont traditionnellement porté sur les interprétations que les sciences de la vie font des différences interindividuelles. Les biosciences ont en effet souvent été accusées de pratiquer un réductionnisme biologique et de mettre davantage l'accent sur l'inné que sur l'acquis, contribuant ainsi à la naturalisation des inégalités et à la diffusion d'une idéologie des dons biologiquement fondée. Dès les années 1980, certains spécialistes des sciences de la vie, comme JeanPierre Changeux, se sont efforcés de désactiver ces critiques en soulignant l'intrication du biologique et du social (Lemerle, 20I4a). Même s'il demeure aujourd'hui des chercheurs qui affirment le caractère premier des déterminations biologiques et génétiques (Perbal, 20ı I), l'approche multifactorielle intégrant l'étude des relations entre des facteurs bio-psycho-sociaux s'est largement imposée comme la plus légitime. Dans la mesure où les facteurs biologiques/ génétiques et environnementaux sont en continuelle interaction, il ne serait ni possible ni souhaitable de chercher à isoler leurs poids respectifs et de les hiérarchiser (Morel, 20I5). Outre qu'elle désactive, en la reléguant au passé et au dépassé, la critique indissociablement scientifique et politique de réductionnisme biologique, cette mise à distance de la question de la hiérarchisation des ensembles de facteurs bio-psycho-sociaux agissant sur la cognition s'effectue au profit d'une posture pragmatique visant à privilégier la description et l'action aux explications causales et ontologiques (Hackman et Farah, 2009).

L'un des principaux mérites des recherches en NC serait ainsi d'identifier des «problèmes » cognitifs sur lesquels il est possible d’agir. Cette orientation vers l'action se traduit, au sein de l'univers des NC, par l'absence d'une division du travail fortement marquée entre la recherche fondamentale et la recherche appliquée. Un chercheur comme Stanislas Dehaene, professeur au collège de France et personnalité centrale des NC en France, se consacre à la fois à l'étude des bases neurales de la lecture (Dehaene, 2007) et aux applications potentielles de ses recherches dans le domaine des apprentissages scolaires (Dehaene, 20II). En d’autres termes, la recherche fondamentale est présen- 
tée comme débouchant sur des «interventions» susceptibles d’améliorer les performances cognitives, à commencer par celles des enfants, dont le cerveau est plus plastique que celui des adultes. Le crédit accordé aux préconisations des chercheurs en NC est par ailleurs d'autant plus grand que ceux-ci sont, expériences à l’appui, particulièrement impliqués dans l'administration de la preuve de leur efficacité. Les enquêtes longitudinales (avec groupe témoin) montreraient par exemple que les méthodes pédagogiques centrées sur le décodage des correspondances grapho-phonémiques sont les plus efficaces (Torgensen, 2002). De même, les expériences en laboratoire révèleraient l'existence de styles d'apprentissage universels et prouveraient l'efficacité des remédiations informées par les NC sur de faibles lecteurs en révélant, par exemple, l'activation de zones cérébrales associées à la lecture auparavant non activées (Eden et al., 2004). Si les recommandations des spécialistes des NC dans le domaine des apprentissages sont rarement novatrices, l'administration de la preuve de leur efficacité par la statistique et, surtout, par la mise en évidence des effets des rééducations sur le cerveau via les techniques visuelles d’analyse de l’activité cérébrale (Dumit, 2005), est, elle, relativement innovante et, en partie au moins, à l'origine de la force de conviction actuelle des NC. L'enjeu est important puisqu'il s'agirait, comme le dit Stanislas Dehaene à propos d'expériences sur la mémoire, d'élaborer une pédagogie fondée sur la preuve («evidence-based education», voir Davies, 1999) dont l'efficacité serait «démontrée expérimentalement et non plus simplement hypothétique ${ }^{2} »$.

L'espoir suscité par de possibles interventions est donc sous-tendu par un discours aux accents anti-déterministes - ce qu'il ne faut pas confondre avec la négation de tout déterminisme biologique ou génétique - qui se fonde sur la connaissance des bases neurales de la lecture, de la plasticité du cerveau et de l'efficacité «expérimentalement avérée » de certaines remédiations. De ce fait, les spécialistes des NC considèrent qu’il sont légitimement fondés à intervenir dans les débats sur certains problèmes sociaux et à orienter l'action publique. Ainsi, dans le domaine des apprentissages scolaires, soulignent-ils l'importance

2 Cours au Collège de France, 3 février 2015: <http://www.college-de-france.fr/site/stanislasdehaene/course-2015-02-03-09h30.htm>, consulté le I8 juillet 2015. 
des politiques de prévention ciblant les enfants «à risque » afin de compenser un faible niveau de conscience phonologique ou d'attention susceptible de leur être préjudiciable tout au long de leur vie et, en particulier, lors de leurs études. Telles qu'elles sont présentées par les chercheurs eux-mêmes, les NC seraient donc associées à un projet de transformation sociale scientifiquement informé qui, dans le domaine des apprentissages scolaires par exemple, permettrait de réduire les inégalités. Comme beaucoup d’autres savants avant eux, les spécialistes des NC peuvent être considérés comme des réformateurs particulièrement influents.

Il n'incombe pas au sociologue, surtout quand il ne dispose que de peu de recul historique, de se prononcer sur la capacité réelle des NC à peser efficacement sur des problèmes sociaux comme l'échec scolaire. Il lui revient, en revanche, de restituer la production discursive des scientifiques qui conditionne la réception de leurs théories auprès d'un large public. De ce point de vue, il est incontestable que les NC sont, au moins en partie, parvenues à faire vaciller certaines des critiques qui entravaient leur diffusion ainsi qu'à se faire reconnaître comme une discipline matérialiste, pragmatique, assimilatrice et progressiste, orientée vers l'action et porteuse d'espoirs.

\subsection{DU NORMAL AU PATHOLOGIQUE Un champ d'intervention élargi}

Si les NC ont avant tout pour ambition d'étudier les relations entre la cognition et les cerveaux de sujets «normaux», elles se sont également spécialisées dans l'étude de certaines pathologies empêchant le développement des capacités cognitives. Ainsi, les difficultés scolaires de certains enfants seraient-elles imputables à des «troubles spécifiques des apprentissages » (TSA), parfois subsumés dans la métacatégorie «galaxie des “dys" » (Habib, 20I4) : dyslexie, dysorthographie, dyscalculie, dysphasie, dyspraxie, hyperactivité, précocité intellectuelle, etc. Ces TSA sont particulièrement intéressants à étudier pour l'ensemble de la communauté des neuroscientifiques car ils permettent de mieux comprendre, via la comparaison, certains mécanismes neuraux de la cognition: le fonctionnement particulier du cerveau du dyslexique éclaire ainsi celui d'un normo-lecteur. 
Tableau I. La constellations des «DYS »

\begin{tabular}{|l|l|c|}
\hline \multicolumn{1}{|c|}{ TROUBLES } & \multicolumn{1}{|c|}{ SYMPTÔMES } & PRÉVALENCE \\
\hline Dyslexie & $\begin{array}{l}\text { Difficultés d'apprentissage du } \\
\text { langage écrit }\end{array}$ & 3 à II\% \\
\hline Dysorthographie & $\begin{array}{l}\text { Difficultés à orthographier les } \\
\text { mots }\end{array}$ & Données non disponibles \\
\hline Dysphasie & $\begin{array}{l}\text { Difficultés d'apprentissage du } \\
\text { langage oral }\end{array}$ & I à 2\% \\
\hline Dyscalculie & $\begin{array}{l}\text { Performances très basses en } \\
\text { mathématiques }\end{array}$ & 3 à 6\% \\
\hline Dyspraxie & $\begin{array}{l}\text { Difficultés à planifier et à coor- } \\
\text { donner les gestes }\end{array}$ & 5 à $7 \%$ \\
\hline $\begin{array}{l}\text { Syndrome hyperkénétique } \\
\text { /hyperactivité (TDAH) }\end{array}$ & $\begin{array}{l}\text { Problèmes de concentration } \\
\text { avec ou sans hyperactivité }\end{array}$ \\
\hline $\begin{array}{l}\text { Précocité } \\
\text { (enfants surdoués) }\end{array}$ & $\begin{array}{l}\text { Dyssinchronie entre l'âge affectif } \\
\text { et l'âge intellectuel, difficultés } \\
\text { d'insertion scolaire }\end{array}$ \\
\hline Syndrome hémisphère droit & $\begin{array}{l}\text { Difficultés d'organisation spa- } \\
\text { tiale, comportements asociaux }\end{array}$ & Données non disponibles \\
\hline
\end{tabular}

Au-delà de son intérêt scientifique intrinsèque, l'étude des TSA a eu pour conséquence non négligeable de mobiliser encore davantage la communauté médicale dans les recherches en NC. De ce fait, le projet de transformation sociale par les NC a été renforcé par l'activation du relais important que constituent les professionnels de santé. On compte actuellement de plus en plus de neurologues, pédiatres, pédopsychiatres, médecins scolaires et, surtout, de professions paramédicales (orthophonistes, psychomotriciens, ergothérapeutes, orthoptistes) impliqués dans l'étude des TSA ainsi que dans l'élaboration et la mise en œuvre de traitements («rééducations », «entraînements ») ciblant ce type de troubles. Progressivement constitués en problème de santé publique, les TSA ont donné lieu, dès le début des années 2000, à plusieurs «Plans d’action» (cosignés par les ministères de l'Éducation nationale et

3 Dyslexie (Habib, 2014), dysorthographie (Inserm, 2007), dysphasie (Ringard, 2000), dyscalculie (Inserm, 2007), dyspraxie (voir Inserm: <http://www.inserm.fr/thematiques/neurosciences-sciencescognitives-neurologie-psychiatrie/dossiers-d-information/troubles-des-apprentissages-les-troublesdys >), hyperactivité (DMS-IV, 2002) et précocité (Catheline, 2012). 
de la Santé) visant à améliorer la détection et la prise en charge des enfants atteints par ces troubles. Ces plans d'action, associés à l'obtention d'importants moyens financiers, ont été des leviers particulièrement puissants pour développer l'action des NC dans le domaine des difficultés d'apprentissage scolaire. Consécutivement, de nombreux services hospitaliers se sont spécialisés dans la prise en charge des TSA, la diffusion des connaissances en NC (en particulier en direction des orthophonistes) a été organisée dans des réseaux de soin et les formations sur les TSA se sont multipliées en direction des enseignants, notamment sous l'impulsion des médecins scolaires.

Dans la mesure où l'inscription des TSA dans le registre de la pathologie est indissociable de l'hypothèse de leur origine organique et génétique, i.e. de l'identification d'une cause «première » biologique (Ramus, 2005), l'ampleur inédite de la médicalisation des difficultés d’apprentissage (voir les taux de prévalence dans le tableau précédent ${ }^{4}$ ) fait néanmoins planer la menace d’une réactivation des critiques de biologisation du social (Fijalkow, 2000; Inizan, 1998) allant à l'encontre des efforts produits par les chercheurs en NC pour échapper aux débats sur la hiérarchisation des facteurs bio-psycho-sociaux impliqués dans les apprentissages. Cette tension a été en partie résolue grâce à un travail d'euphémisation du caractère pathologique de ces troubles et à une prise de distance avec les positions jugées trop organicistes, y compris celles formulées par des chercheurs en NC ou par des médecins. Le terme même de «trouble» (en lieu et place de «pathologie» ou de «maladie ») est censé mettre davantage l’accent sur la symptomatologie des TSA que sur leur origine pathologique. Les chercheurs ont par ailleurs insisté sur la banalité et le caractère le plus souvent bénin de la plupart de ces troubles: de nombreux enfants atteints de TSA auraient du mal à apprendre à lire comme beaucoup d’autres ont des problèmes de vue. Dans un cas comme dans l'autre, l'important serait de ne pas ignorer le problème et de mettre en place les solutions efficaces disponibles (Ramus, 2005). En outre, à la différence

4 Bien qu'il ne soit pas possible de déterminer le nombre d'enfants atteints par ces troubles en procédant à une simple addition des taux de prévalence, un individu pouvant être atteint par plusieurs troubles, les chiffres du tableau indiquent clairement l'ampleur de la médicalisation. On peut en effet penser qu'entre 10 à $25 \%$ des enfants sont atteints par un ou plusieurs des troubles présentés dans le tableau. 
du retard global (déficience intellectuelle), diagnostic pendant longtemps abondamment utilisé, sous sa forme savante ou profane, pour expliquer les difficultés scolaires de certains élèves, les TSA supposent la possession d'une intelligence «normale »: ainsi, l'enfant dyslexique obtient-il des scores très faibles dans les parties des tests d'intelligence qui portent sur les compétences langagières, mais des résultats «normaux» dans les parties qui mesurent sa capacité à raisonner ou à manipuler des objets. On oublie trop souvent que, pour comprendre les usages sociaux de ce type de catégories diagnostiques, la mise en lumière par les NC de l'intelligence normale des individus atteints de TSA est au moins aussi importante que l'identification de certains dysfonctionnements cognitifs. En vue d'euphémiser la médicalisation, les chercheurs en NC ont enfin tenté de dissocier le traitement des TSA des représentations traditionnellement associées à l'intervention médicale. À l'exception notable du traitement pharmacologique de l'hyperactivité, les TSA, à commencer par les troubles spécifiques du langage les plus courants comme la dyslexie, appelleraient des rééducations pédagogiques pouvant être mises en œuvre par des orthophonistes, voire par des enseignants ayant suivi une formation (Ramus, 2005), comme c'est d'ailleurs le cas dans certains pays anglo-saxons (Woollven, 20I2). En somme, le travail de construction des TSA en problème de santé publique permet aux chercheurs en NC de souligner l'urgence de la reconnaissance de ce genre de «troubles» et, partant, des théories diagnostiques qui les sous-tendent, tout en demeurant à la frontière du médical et du pédagogique et en se protégeant ainsi des traditionnelles critiques visant la médicalisation de l'échec scolaire.

En définitive, dans un contexte où le système éducatif français est perçu comme miné par un échec scolaire persistant et des inégalités croissantes (OCDE, 20I4), les chercheurs en NC ont profité de l'essoufflement, supposé ou réel, d'interprétations de l'échec scolaire construites dans les années 1960 (sociologiques, psychanalytiques) pour œuvrer à la construction de solutions présentées comme des alternatives porteuses d’espoir. Fondant leur démarche sur le dépassement de certains clivages qui structuraient l'espace des prises de position sur les difficultés d’apprentissage (inné/acquis, biologique/social, normal/pathologique), ils sont parvenus à s'imposer auprès d'un large public comme des scientifiques 
engagés dans une résolution pragmatique de l'échec scolaire et dégagés des a priori pédagogiques, idéologiques ou politiques. Outre les intermédiaires classiques, comme les journalistes ou les éditeurs 5 , ils ont par ailleurs fédéré autour d'eux un réseau d'institutions et de professionnels de santé susceptibles de diffuser et de mettre en œuvre ces alternatives. La construction des TSA en troubles de santé publique leur a enfin permis de faire jouer certains leviers de l'action publique et de revendiquer l'urgence du développement des recherches en $\mathrm{NC}$ et de la mise en œuvre des traitements des difficultés d’apprentissage qui en découlent. L'entreprise de légitimation d'une «nouvelle» réponse aux difficultés d’apprentissage a néanmoins bénéficié de l'existence d’autres relais susceptibles de soutenir et de diffuser les analyses et les recommandations des NC. Deux d'entre eux seront maintenant examinés, à savoir, d'une part, les hommes politiques, hauts fonctionnaires et experts chargés d'élaborer et de mettre en œuvre une réforme pédagogique, et, d’autre part, les parents d'élèves en difficulté dans le cadre scolaire.

\section{NEUROSCIENCES COGNITIVES ET RÉFORMES PÉDAGOGIQUES}

L’approche des difficultés d'apprentissage par les NC s'est d’abord trouvée, à partir des années 1990, en phase avec le travail de redéfinition du problème de l'échec scolaire entrepris par certains experts (universitaires, spécialistes de l'éducation exerçant dans les organisations internationales), hauts fonctionnaires ou hommes politiques (Morel, 2014). Les constats alarmistes sur les performances du système éducatif français (augmentation des inégalités, niveau très bas et en baisse des élèves les plus faibles - OCDE, 20I4) ont conduit à redéfinir les modalités de la démocratisation scolaire et de la lutte contre l'échec scolaire. À l'ambition prioritaire de faire accéder l'ensemble d'une classe d'âge à des études de plus en plus longues et à des titres scolaires de plus en plus élevés (80\% d'élèves titulaires d'un bac, 50\% titulaires d'un diplôme du supérieur), s'est substituée celle d'assurer à l'ensemble des élèves une meilleure maîtrise des savoirs fondamentaux au cours de la scolarité obli- 
gatoire et, plus particulièrement, à l'école primaire. Apprendre aux élèves à être plus performants dans certaines compétences de bases (lire, écrire, compter) qui conditionnent toute la scolarité est devenu la priorité affichée des gouvernements successifs, quelle que soit leur couleur politique. Outre le déplacement du centre de gravité du problème de l'échec scolaire vers le premier degré et le recentrement sur les savoirs fondamentaux, la différenciation des apprentissages a été le second mot d’ordre de la réforme pédagogique. Depuis le début des années 2000, l’idée que le collège unique français n’individualise pas suffisamment les enseignements s'est largement imposée, tout comme le fait que l'échec scolaire résulte avant tout de la somme des défaillances individuelles d'élèves à besoins éducatifs particuliers. Ces deux grandes orientations de la réforme pédagogique sont en phase, selon des modalités qu'il convient de définir, avec l’approche des difficultés d’apprentissage par les NC.

\section{I. UNE SCIENCE DES OPÉRATIONS COGNITIVES ÉLÉMENTAIRES}

Jusquaux années 1980, les sciences sociales sont parvenues à imposer une représentation dominante de l'échec scolaire comme phénomène social qui trouve son origine dans le rapport distant de certains élèves (en grande majorité issus des milieux économiquement et culturellement défavorisés) à la culture, à la forme ou aux normes scolaires. Cette explication prend d'autant plus de poids que les élèves avancent dans leur scolarité et qu'ils se retrouvent confrontés à des apprentissages complexes supposant une socialisation antérieure à la culture savante et lettrée. Et ce n'est sans doute pas un hasard si Les Héritiers (Bourdieu et Passeron, 1964), ouvrage fondateur de cette approche des inégalités scolaires, est consacré à l'analyse du rapport des étudiants de lettres à la culture scolaire et à la culture libre.

Dans le contexte des années 1990, cette interprétation de l'échec scolaire sous sa variante vulgarisée du handicap socioculturel (les familles de milieux populaires ne donnent pas à leurs enfants ce dont ils ont besoin pour réussir leur scolarité) a fait l'objet de nombreuses critiques, dont certaines issues des sciences sociales. Elle fut, d'une part, accusée de véhiculer une forme de déterminisme sociologique et d'exonérer le fonctionnement social de l'École et les pratiques 
de ses professionnels de toute responsabilité dans la production de ces inégalités. À une période où les explications «classistes» étaient particulièrement mises en cause, on accusa, d'autre part, cette approche des inégalités scolaires de ne pas expliquer «la grande diversité des performances et des trajectoires scolaires d'une population appartenant à un même "milieu social" ou à une même "culture" et ceci dans des conditions de vie semblables » (Rochex, 2000). Enfin, on lui reprocha de ne pas rendre compte du fait que la plupart des enfants issus des classes populaires réussissent à être performants dans les apprentissages fondamentaux lors des premières années de la scolarité. Une question demeurait donc intacte: comment expliquer qu'une minorité non négligeable d'enfants ( 15 à $20 \%$ des élèves d'une classe d'âge, majoritairement issus des milieux défavorisés) soit en difficulté dans les apprentissages fondamentaux?

C'est au cours de ces années que prit forme le tournant cognitiviste qui se fixa pour objectif d'ouvrir la boîte noire des apprentissages fondamentaux et d'expliciter les processus cognitifs engagés dans l’apprentissage de ces compétences de base dont l'acquisition détermine l'ensemble de la scolarité. Si les sciences sociales contribuèrent à ce champ d'étude (Bonnéry, 2007; Lahire, 1993; Rochex et Crinon, 20I ; Terrail, 20I3), c'est bien la psychologie cognitive qui s'imposa comme la discipline de référence: cette discipline étudiait depuis plusieurs décennies les processus cognitifs élémentaires et prônait elle-même un recentrage sur les apprentissages fondamentaux, ses représentants occupaient des places centrales dans les institutions en charge de diffuser les bonnes pratiques pédagogiques (comme le défunt Observatoire national de la lecture) et la robustesse de ses résultats fut renforcée par les apports des neurosciences à partir des années 1990. Les NC bénéficiaient enfin de la plus grande légitimité des sciences de la vie et de la psychologie dans le domaine de l'identification des «problèmes» des jeunes enfants (Lignier, 2015).

Outre leur capacité à décrire les processus cognitifs impliqués dans les apprentissages fondamentaux (différents types de mémoire, conscience phonologique, attention, etc.) et à en révéler la réalisation physique et matérielle dans le cerveau, les chercheurs en NC parvinrent également à s'imposer, au détriment des pédagogues socioconstructivistes ou des psychanalystes (IGAS/IGEN, 2002), comme 
les détenteurs de solutions efficaces aux problèmes de l'échec précoce. Les chercheurs en NC purent notamment faire valoir leur objectif explicite de résolution des difficultés scolaires (leur action étant évaluée à l'aune des progrès scolaires effectués par les élèves), là où leurs concurrents (comme les psychanalystes) refusaient une telle évaluation objectivante de leur travail de remédiation et, partant, étaient dans l'incapacité de répondre aux nouvelles exigences de pouvoirs publics conditionnant leurs dépenses dans le domaine de la lutte contre l'échec scolaire à l'administration de la preuve de l'effet des actions entreprises sur le niveau scolaire. Ainsi, dans le domaine de la lecture, où la compréhension des textes et la prise en compte du plaisir de lire ont été longtemps privilégiés, les NC firent de l'intensification des exercices visant à améliorer la conscience phonologique (familiarité avec les structures élémentaires du langage) et, en particulier, du travail de décodage grapho-phonémique, la condition sine qua non de l'amélioration des performances, prouvée en laboratoire, de tous les élèves dans le domaine de la lecture. Cependant, loin de se limiter à ces recommandations pouvant être perçues par certains comme rétrogrades, les spécialistes des NC, formulant des recommandations proches de celles des pédagogies nouvelles, suggérèrent aussi des relations maîtres/élèves fondées sur la bienveillance (absence de peur de la sanction, évaluation formative), sur l'activité (Freeman et al., 20I4) et la participation (la méthode de Maria Montessori étant souvent prise en exemple comme technique favorisant l'attention des élèves). Ces préconisations, loin d'être novatrices pour la plupart, tirent une grande partie de leur pouvoir de conviction du type d’administration de la preuve qui leur est associé : elles seraient, pour la première fois, expérimentalement démontrées grâce à des expériences sur des cerveaux humains ou animaux.

Bien que critiquées comme réductrices et déconnectées des situations réelles d'apprentissage au sein même des sciences cognitives (voir par exemple les travaux du psychologue Roland Goigoux [2013], très diffusés dans les institutions en charge de la formation des enseignants du premier degré), ces recommandations furent très vite reprises à leur compte par beaucoup de hauts fonctionnaires et d'hommes politiques engagés dans la réforme pédagogique. Après Jack Lang, Ségolène Royal et Bernard Kouchner, qui avaient relayé, à la fin des années 1990 et au début des années 2000, les recommandations péda- 
gogiques formulées par les spécialistes des NC, Gilles de Robien, alors ministre de l'éducation, déclarait ainsi en 2005 : «La méthode globale a été abandonnée en théorie par les nouveaux programmes. Mais dans la pratique, même si la méthode globale pure a disparu depuis longtemps, il reste des méthodes "à départ global”. Ces méthodes font commencer l’apprentissage de la lecture par une approche globale pendant plusieurs semaines, pour n'en venir qu'ensuite à la découverte des syllabes. Les neurosciences permettent aujourd'hui de dire que cette approche est mauvaise ».

Les recommandations des NC furent également reprises à leur compte par les hauts fonctionnaires du ministère de l'Éducation nationale et intégrées en 20 Io au nouveau référentiel de compétences à acquérir par les professeurs (le référentiel indique ainsi pour la compétence $\mathrm{n}^{\circ} 6$, «Prendre en compte la diversité des élèves», que les enseignants doivent connaître «les mécanismes de l'apprentissage dont la connaissance a été récemment renouvelée, notamment par les apports de la psychologie cognitive et des neurosciences $»^{6}$ ).

Par ailleurs, dans le domaine de la lutte contre l'échec scolaire, les chercheurs NC s'accommodèrent de la diffusion d'une métacatégorie d'intervention publique comme celle d'«élèves à risque», dont ils font eux-mêmes un usage assez intensif, et qui est en accord avec leur approche pragmatique des difficultés d'apprentissage dont un des objectifs est de reléguer au second plan les polémiques sur la hiérarchisation des facteurs à l'origine des difficultés d'apprentissage au profit d'une intervention efficace. Les recherches en NC mettent ainsi en avant la nécessité de traiter précocement les enfants «à risque » sans préciser les contours de cette métacatégorie. Sont considérés comme tel les élèves de milieux très défavorisés, de parents souffrant de TSA ou ceux, plus simplement, qui affichent un faible niveau de conscience phonologique lorsqu'on leur fait passer un test précoce. Le danger d'étiquetage associé à une telle catégorisation est relativisé, voire dénié (Ramus, 20I I) au profit de la promotion du paradigme actuellement dominant en santé publique, à savoir celui inspiré de l'épidémiologie. 


\subsection{RÉPONSES CIBLÉES ET MAXIMISATION DES POTENTIELS COGNITIFS}

La redéfinition de l'échec scolaire à partir des années 1990 a conduit à une seconde inflexion des politiques éducatives: la remise en cause de l'uniformité de l'École française. Claude Thélot affirme ainsi dans le rapport issu de la Consultation nationale sur l'École en 2004: «Une École de masse ne doit pas être, au risque d'être aussi une École de l'exclusion, une École de l'uniformité » (Thélot, 2004). En somme, le diagnostic est le suivant: si l'école unique fonctionne mal en France, c'est parce que, prisonnière du dogme de l'unification des méthodes, des objectifs et des contenus, elle est incapable de différencier les apprentissages en fonction des besoins singuliers des élèves. C'est aussi parce qu'elle aurait trop cherché, comme dans le cas de la politique des zones d'éducation prioritaire, à appréhender l'éducation au niveau de «collectifs abstraits » au lieu de privilégier l'intervention au niveau individuel (ibid.). S'inspirant de l'«intégration individualisée» mise en ouvre dans les pays scandinaves ou asiatiques, l'École française devrait, au contraire, accentuer la différenciation des enseignements. Elle devrait également appréhender l'échec scolaire comme la somme de défaillances individuelles d'élèves «à besoins éducatifs particuliers » (OCDE, 2003), dont les difficultés sont imputables à des causes variées et, partant, non réductibles aux facteurs sociaux. L'important serait donc de reconnaître que, de même qu'il existe une «pluralité des excellences», il existe une pluralité des échecs, et que l'important n’est pas de hiérarchiser les causes des difficultés en fonction de leur poids respectif, mais d'être en mesure de proposer une gamme d’enseignements adaptés aux besoins spécifiques des élèves. Cette évolution s'est par exemple traduite par une transformation des modes ciblages des élèves en difficulté dans le cadre des politiques d'éducation prioritaire. Comme le rappelle Jean-Yves Rochex, «le cadre de référence des politiques d'éducation prioritaire, mais aussi des politiques scolaires génériques, n’est plus le rapport des différents milieux sociaux au système éducatif, mais l’adaptation de celui-ci à la diversité des individus » (Rochex, 20ıо). 
Cet infléchissement du traitement de l'échec scolaire constitue un terrain d'intervention favorable pour les chercheurs en NC. D’abord parce que ces derniers défendent eux-mêmes l'individualisation des apprentissages. S'ils réfutent l'idée d'une profusion des styles d’apprentissage (Pashler et al., 2008) et insistent sur le fait que certaines méthodes d'apprentissage sont universellement efficaces, ils soulignent en revanche, à partir d'observations du fonctionnement du cerveau, que l'attention portée par l'adulte à l'enfant (Egyed et al., 20I3), la mise en activité des élèves par le maître ainsi que la prise en compte des différents niveaux cognitifs des élèves au sein d'une classe - qui dépendent évidemment du nombre d'élèves à laquelle s’adresse un enseignant - sont déterminants pour les apprentissages scolaires.

Dans la mesure où elles produisent une abondance de catégories diagnostiques (la «galaxie des “dys” » par exemple), dont certaines semblent calquées sur les catégories scolaires (dysorthographie, dyscalculie), les NC contribuent également au processus de différenciation des apprentissages par extension et diversification du répertoire des élèves à «besoins éducatifs particuliers ». Ainsi, de même que les élèves issus de milieux socialement très carencés ou nouvellement arrivés en France gagneraient à recevoir un enseignement spécifique, de même les élèves dyslexiques, dysorthographiques, dyscalculiques, dysphasiques, dyspraxiques, hyperactifs, intellectuellement précoces, etc. seraient d’autant plus performants dans les apprentissages scolaires qu'on leur proposerait des enseignements ajustés à leurs spécificités cognitives. Cet éclatement des modes de ciblage, loin d'être le signe d'une fragmentation, potentiellement infinie, des politiques de lutte contre l'échec scolaire, est majoritairement considérée par les responsables éducatifs comme l’indice des efforts réalisés pour adapter le système scolaire français à la diversité des besoins des élèves.

Enfin, parce qu'elles adhèrent, du fait de leur ancrage dans les biosciences, à l'«évidence d d'une différenciation des capacités cognitives en fonction de l'appareillage génétique et biologique des individus - tout en relativisant tout déterminisme en la matière -, les NC sont en phase avec la remise en cause actuelle, sous une forme euphémisée, de l'égalitarisme qui aurait prévalu dans la première phase de démocratisation scolaire. Elles contribuent ainsi à renforcer 
la logique actuellement dominante ${ }^{7}$ selon laquelle l'efficacité de l'École devrait désormais être évaluée en fonction de sa capacité à maximiser le potentiel d'élèves aux capacités cognitives biologiquement et socialement différenciées.

Pour toutes les raisons qui viennent d'être évoquées, on peut dire que le succès de l'importation des NC dans le domaine des apprentissages scolaires tient, au moins pour une part, au fait que s'y réfractent, plus que dans d'autres disciplines, les représentations dominantes, produites dans l'univers politique et scolaire, de ce que veut actuellement dire «lutter contre l'échec scolaire». Cette réfraction n'est pas seulement le produit d'une convergence asymptotique à un instant t, elle est aussi la conséquence de l'appropriation des recherches en $\mathrm{NC}$ dans les milieux politiques et pédagogiques.

\section{USAGES DES «TROUBLES SPÉCIFIQUES DES APPRENTISSAGES» PAR LES PARENTS D'ÉLĖVES EN DIFFICULTÉ}

L’analyse des usages sociaux des NC serait incomplète si elle n'étudiait pas le rôle central désormais joué par les parents d'élèves en difficulté, tout particulièrement dans l'appropriation et la diffusion des TSA. Jusqu'à récemment, les travaux issus des sciences sociales ont souvent fait des individus ou des groupes sociaux confrontés à la médicalisation les cibles passives d'un processus sur lequel ils n'avaient que peu de contrôle. Dans le sillage de Michel Foucault (1988, 2003 [1973-1974], 2004 [1978-1979]), la médicalisation d'un nombre croissant de problèmes humains a ainsi été appréhendée comme un phénomène impulsé d'«en haut» par l'État et/ou par certaines fractions des classes dominantes conduisant, grâce à l'intervention d'une série de professionnels (professions médicales et paramédicales, psychologues, travailleurs sociaux, etc.), à un contrôle des populations déviantes.

$7 \quad$ Elle le fut aussi dans la première moitié $d u x x^{e}$ siècle avant que la notion d'aptitudes, qui était au fondement du projet de démocratisation dans les années 1950, soit la cible de nombreuses critiques, notamment issues des sciences sociales. 
Ce type d'analyse prend toute sa pertinence dans un contexte où le registre médico-psychologique est monopolisé par des élites politiques ou professionnelles, qui en maîtrisent les usages. Mais, on peut s'interroger sur sa validité lorsque ce registre se propage, sous des formes vulgarisées, dans de nombreuses régions de l'espace social. Or, on assiste, depuis une vingtaine d'années, à une diffusion très rapide des connaissances sur les troubles spécifiques des apprentissages auprès d'un large public, qui invite à un renouvellement des interprétations du rapport des familles à la médicalisation, en particulier dans le cas de l'échec scolaire. Désormais, un nombre croissant de parents, appartenant pour la plupart aux classes moyennes et supérieures, loin de ne faire que subir l'étiquetage médico-psychologique des difficultés scolaires de leur(s) enfant(s), se mobilisent pour la reconnaissance des diagnostics les moins stigmatisants (pour l'enfant, mais aussi pour ses parents) et les plus compatibles avec la poursuite d’une scolarité «normale». Elles mobilisent les diagnostics médicaux pour faire valoir leur point de vue dans l'univers scolaire, parfois contre les enseignants. Elles interviennent également de plus en plus dans les controverses scientifiques autour de la médicalisation de l'échec scolaire et pèsent sur l'issue des concurrences entre approches rivales.

Les analyses qui suivent s'appuient sur des données recueillies lors d'une série de vingt-trois entretiens réalisés en 2008 auprès de parents rencontrés dans un service hospitalier spécialisé dans le diagnostic et le traitement des TSA. Si elle ne peut être généralisée, l’analyse met cependant en lumière, sous une forme idéale-typique, les principales logiques à l'œuvre lors de l’appropriation par les parents des explications des difficultés d’apprentissage par les NC.

\section{I. OPTIMISER LA SCOLARITÉ}

Les entretiens mettent clairement en évidence qu'un des objectifs centraux des mobilisations parentales est de maintenir scolairement à flot leurs enfants malgré leurs difficultés. Il s’agit d’abord de peser sur les représentations que les enseignants se font des «problèmes » de leur enfant. Les parents essayent de substituer aux catégories traditionnelles de l'entendement professoral (Bourdieu et Saint-Martin, I975) qui tendent à imputer les difficultés de l'en- 
fant à un manque de travail et de motivation ou aux dysfonctionnements de la cellule familiale, une perception à la fois plus objective et compréhensive reconnaissant l'existence de difficultés «réelles» sur lesquelles l'enfant et ses parents n’ont que peu de prise. Les parents s'appuient sur les préconisations des professionnels de santé pour obtenir certains aménagements scolaires (refus ou obtention d'un redoublement, saut de classe, choix de classe ou d'établissement, adaptations pédagogiques, obtention d'une auxiliaire de vie scolaire, temps supplémentaires aux examens, etc.). Néanmoins, tous les diagnostics ne se valent pas pour les parents. Certains, comme ceux qui imputent les difficultés de l'enfant à des difficultés psychoaffectives, maintiennent, voire accentuent leur sentiment de culpabilité. Si, pour le bien de leur enfant, beaucoup d'entre eux acceptent de consulter un «psy», ils le font souvent avec beaucoup de résistances. Les parents et, en particulier, les pères formulent, à quelques exceptions près, des critiques très virulentes à l'encontre des «psys », jugés intrusifs, fous, culpabilisants, irrationnels, crédules, dangereux et, surtout, inefficaces. L'interprétation psychologisante est d’autant plus critiquée qu'elle paraît coupée du versant scolaire des difficultés de l'enfant ou difficilement mobilisable dans les relations avec le corps enseignant. À l'inverse, les interprétations qui attribuent les difficultés de l'enfant à des troubles du langage ou de la motricité fine sont mieux reçues par les parents. Débouchant sur des prises en charge en orthophonie, en psychomotricité ou en ergothérapie, voire à des reconnaissances de handicap, elles sont perçues comme moins stigmatisantes et culpabilisantes, plus en prise avec les questions scolaires et davantage mobilisables pour suggérer aux enseignants l'existence de troubles «réels» indépendants de la volonté de l'enfant ainsi que la nécessité d’adaptations pédagogiques. Les nombreux «bilans » effectués dans le cadre de ces rééducations et les tests de Q.I. réalisés par les psychologues rassurent enfin, dans la grande majorité des cas, les parents sur l'«intelligence» de leur enfant.

Comprendre l'appropriation des troubles spécifiques des apprentissages par les parents suppose donc de la rapporter aux intérêts qui la sous-tendent. Cette appropriation ne repose pas que sur une évaluation de la pertinence scientifique du diagnostic et de son caractère ajusté à la perception des difficultés de l'enfant 
par les parents. Un diagnostic vaut aussi pour tout ce qu'il permet de croire, de faire, d'obtenir. De ce point de vue-là, les TSA paraissent, au moins à court terme, plus avantageux pour les familles que d'autres diagnostics (Morel, 2012).

\subsection{MOBILISATIONS COLLECTIVES DES ASSOCIATIONS DE PARENTS D'ENFANTS «DYS»}

Si l'enquête ethnographique saisit avant tout les mobilisations individuelles des parents d'enfants en échec, il faut néanmoins rappeler que les associations de parents jouent, à un autre niveau, un rôle décisif dans le processus de diffusion des NC. L'action des associations de parents d'élèves «dys» ou «intellectuellement précoces », de plus en plus nombreuses, s’est avérée décisive pour la reconnaissance des troubles spécifiques des apprentissages. Agissant comme des groupes de pression, ces associations ont un répertoire d’actions assez étendu.

Elles prétendent d'abord être des centres ressources pour les parents : elles informent leurs membres des avancées de la recherche en NC, les conseillent, les mettent en relation les uns avec les autres lors de réunions ou dans le cadre de forums sur Internet. Situant une partie de leur activité sur le versant de la dénonciation morale, elles agissent au nom de «victimes », c'est-à-dire des enfants (et de leur famille), dont les troubles spécifiques des apprentissages ne seraient pas reconnus ou mal interprétés. De concert avec certains acteurs du monde scientifique ou médical, elles contribuent à la recension des recherches sur les différents troubles et à leur vulgarisation via leur site Internet ou grâce à la publication d'ouvrages destinés au grand public.

Elles nouent ensuite des liens très étroits avec les experts qui occupent des positions-clés dans le domaine qui les concerne: le comité scientifique de la Fédération française des «dys » (FFDys) est ainsi présidé par Jean-Charles Ringard, l'inspecteur d'académie ayant rédigé le rapport sur les enfants dysphasiques et dyslexiques, et compte dans ses rangs Franck Ramus, un des chercheurs français les plus impliqués dans la recherche en NC sur les TSA et de nombreux médecins et psychologues spécialisés dans les troubles spécifiques des apprentissages. Les responsables des associations sont également présents dans les groupes de travail constitués pour la rédaction des rapports officiels (le groupe réuni dans le cadre 
du rapport commandé à Ringard (2000) comportait cinq représentants d'associations de parents «dys»). Les revendications des associations s'adressent enfin à la classe politique qu'elles essayent de mobiliser autour de la cause des «dys».

Les associations de parents d’enfants «dys » travaillent également à la reconnaissance de la spécificité des élèves atteints de TSA. Ces stratégies distinctives poussent les associations à rappeler l'irréductible différence, expérimentalement démontrée, entre ce type de troubles et les différentes formes de psychopathologies ou de déficiences intellectuelles. Elles mettent en œuvre un travail de veille visant à dénoncer tout brouillage des frontières entre les différents diagnostics, que ce soit au niveau théorique ou au niveau des dispositifs d'enseignement. Elles militent ainsi pour que les dispositifs accueillant des élèves à besoins éducatifs particuliers évitent l'«amalgame» entre les enfants atteints de troubles spécifiques des apprentissages et ceux dont les difficultés sont imputables à d’autres «problèmes » (retard global, psychopathologie ou handicap socioculturel). S'adressant aussi bien aux parents qu'à l'opinion publique, elles effectuent enfin un travail de démarcation entre les handicaps moteurs ou mentaux, qui désignent de véritables déficiences, et celui dont souffrent les enfants «dys», dont la principale raison d'être serait administrative. La différence entre les deux types de handicaps est aussi présentée sous l'angle des pronostics qui leurs sont associés. Alors que le handicap est en général associé à une difficulté définitive ou, au moins, durable, les TSA sont présentés par les associations comme compatibles avec une normalisation de l'existence des patients. Pour montrer que les TSA ne sont pas des troubles «handicapants », les associations organisent des réunions avec des adultes «dys» ayant «réussi leur vie professionnelle». Comme le dit une mère assistant à ces réunions, «ça veut dire qu'on peut être "dys" et s'en sortir». La publication sur leur site Internet de diaporamas où figurent les portraits de «dyslexiques devenus célèbres » (Albert Einstein, Tom Cruise, Bill Gates, Louis Pasteur, Gustave Flaubert, Edgar Poe, Agatha Christie, etc. $\left.{ }^{8}\right)$ participent de la même tentative d'inversion du stigmate et de requalification symbolique des enfants «dys». 
Les associations de parents d'enfants «dys» pèsent donc sur l'issue des concurrences entre disciplines impliquées dans la résolution de l'échec scolaire. Elles ont largement contribué à la promotion des NC, notamment au détriment de la psychanalyse et de certaines approches pédagogiques.

\section{CONCLUSION}

Lapproche sociologique des usages sociaux des biosciences expérimentales en dehors de leur univers d'origine gagne à croiser analyse interne - qui repère ce qui dans la mise en forme scientifique d'une discipline constitue, à un instant t, des prises pour son appropriation par d'autres groupes d'acteurs et analyse externe - qui identifie le degré d’adéquation entre l'approche scientifique étudiée et les attentes de certains groupes d’acteurs dont il s'agit alors d’identifier les croyances, les intérêts ou les stratégies. Parce qu’elle suppose de s'acquitter d'un droit d'entrée coûteux (la familiarisation avec les travaux de la discipline scientifique étudiée), l'analyse interne est parfois négligée par les sociologues, ce qui les conduit à mettre en ouvre une approche critique peu informée, qui s'apparente à une forme de réductionnisme sociologique. Comme j’ai essayé de le montrer ici dans le cas des neurosciences cognitives, il est difficile de comprendre le poids aujourd'hui exercé par cette discipline dans le domaine des apprentissages scolaires sans entrer dans le détail du raisonnement scientifique qu'elle construit sur cette question. La description du traitement des difficultés d'apprentissage par les NC est ainsi un préambule nécessaire à l'analyse de leur large diffusion dans certaines sphères sociales (scolaires, politiques, familiales). Il convient cependant de ne pas réifier la partition entre analyse interne et externe, les disciplines scientifiques ne se construisant pas dans une totale indépendance vis-à-vis des univers non-scientifiques. En ce sens, l’analyse ici proposée pourrait être complétée par une analyse des intermédiaires culturels (journalistes, éditeurs, voir Lemerle, $2014 \mathrm{a}$ et 20I4b) dont le rôle est précisément de créer des relations entre la science et son public (Boltanski et Maldidier, 1977). Il pourrait également être intéressant de montrer, comme Sébastien Lemerle (2014a) a commencé à le faire à propos de certaines figures des biosciences, comment 
les propriétés sociales des scientifiques (à commencer par leur positionnement politique, leurs réseaux de socialisation et de sociabilité) conditionnent à la fois leurs productions scientifiques et l'appropriation de celles-ci par des acteurs partageant leur vision du monde.

\section{BIBLIOGRAPHIE}

BOLTANSKI L. et MALDIDIER P., 1977, La Vulgarisation scientifique et son public, [manuscrit non publié].

BONNÉRY S., 2007, Comprendre l'échec scolaire. Élèves en difficultés et dispositifs pédagogiques, Paris, La Dispute.

BOURDIEU P. et PASSERON J.-C., 1964, Les Héritiers, Paris, Éditions de Minuit.

BOURDIEU P. et SAINT-MARTIN M., 1975, «Les catégories de l'entendement professoral», Actes de la recherche en sciences sociales, I-3, p. 68-93.

CATHELINE N., 2012, Psychopathologie de la scolarité, Paris, Masson.

CHAMAK B. et MOUTAUD B., 2014, Neurosciences et société. Enjeux des savoirs et pratiques sur le cerveau, Paris, Armand Colin.

DAVIES P., 1999, «What Is Evidence-Based Education?», British Journal of Educational Studies, 47-2, p. 108-121.

DEHAENE S., 2007, Les Neurones de la lecture, Paris, Odile Jacob.

-, 20।l, Apprendre à lire, Paris, Odile Jacob.

DONZELOT J., 1977, La Police des familles, Paris, Éditions de Minuit.

DMS-IV, 2002, Manuel diagnostic et statistique des troubles mentaux, Paris, Masson.

DUMIT J., 2005, Picturing Personhood: Brain Scans and Biomedical Identity, Princeton, Princeton University Press.

EDEN G. et al., 2004, «Neural Changes following Clinical Study Remediation in Adult Developmental Dyslexia», Neuron, 44, p. 4lI-422.

EGYED K., KIRÁLY I. et GERGELY G., 2013, «Communicating Shared Knowledge in Infancy», Psychological Science, 24-7, p. 1348-1353. 
FIJALKOW J., 200I, «Dyslexie: le retour», Psychologie et Education, 47, p. III-128.

FOUCAULT M., 1988, «Histoire de la médicalisation », Hermès, 2, p. II-29.

-, 2003 (1973-1974), Le Pouvoir psychiatrique, Paris, Gallimard.

-, 2004 (1978-1979), Naissance de la biopolitique, Paris, Gallimard.

FREEMAN S. et al., 2014, «Active Learning Increases Student Performance in Science, Engineering, and Mathematics», PNAS, III-23, p. 8410-8415.

GARCIA S., 2013, L'École des dyslexiques. Combattre ou naturaliser l'échec scolaire, Paris, La Découverte.

GOIGOUX R., 2013, «Apprentissage de la lecture: opposer méthode syllabique et méthode globale est archaïque», Le Monde, 3I Décembre.

GONON F., KONSMAN J-P. et BORAUD T., 2014, «Neurosciences et médiatisation: entre argumentation de la preuve et rhétorique de la promesse», in CHAMAK et MOUTAUD, 2014, p. 109-133.

HABIB M., 20I4, La Constellation des Dys. Bases neurologiques de l'apprentissage et de ses troubles, Paris, Solal.

HACKMAN D. et FARAH M., 2009, «Socioeconomic Status and the Developing Brain», Trends in Cognitive Sciences, 13-2, p. 65-73.

IGAS/IGEN, 2002, Enquête sur le rôle des dispositifs médico-social sanitaire et pédagogique dans la prise en charge des troubles complexes du langage, rapport de l'Inspection générale des affaires sociales et de l'Inspection générale de l'Éducation nationale.

INIZAN A., 1998, «Et si la dyslexie n'existait pas?», Psychologie et Education, 35, p.33-49.

INSERM, 2007, Dyslexie, Dysorthographie, Dyscalculie. Bilan des données scientifiques, Rapport de l'Inserm.

LAHIRE B., 1993, Culture écrite et inégalités scolaires. Sociologie de l'échec scolaire à l'école primaire, Lyon, PUL.

LEMERLE S., 2014a, Le Singe, le Gène et le Neurone. Du retour du biologisme en France, Paris, PUF.

,$- 2014 b$, «Le neurone des newsmagazines: les neurosciences dans la presse française des années 2000 », in CHAMAK et MOUTAUD, 20I4, p. 8I-I08. 
LIGNIER W., 20I2, La Petite noblesse de l'intelligence. Une sociologie des enfants surdoués, Paris, La Découverte.

-, 2015, «L'identification des enfants. Un modèle utile pour l'analyse des primes socialisations», Sociologie, 6-2, p. 177-194.

MOREL S., 2012, «La cause de mon enfant. Mobilisations individuelles de parents d'enfants en échec scolaire précoce», Politix, 25-99, p. 153-176.

-, 2014, La Médicalisation de l'échec scolaire, Paris, La Dispute.

-, 2015, «Le traitement des difficultés d'apprentissage de la lecture par les neurosciences cognitives : une (re)naturalisation de l'échec scolaire?», communication non publiée, semi-plénière, Congrès de l'Association Française de Sociologie, Université de Versailles Sain-Quentin, 2 juillet.

OCDE, 2003, Déficiences, difficultés et désavantages: statistiques et indicateurs pour l'accès au cursus scolaire et pour l'équité, rapport de l'OCDE.

-, 2014, Principaux résultats de l'Enquête PISA 2012 Ce que les élèves de 15 ans savent et ce qu'ils peuvent faire avec ce qu'ils savent, rapport de l'OCDE.

PASHLER H., MCDANIEL M., ROHRER D. et BJORK R., 2008, «Learning Styles Concepts and Evidence», Psychological Science in the Public Interest, 9-3, p. I05-I19.

PERBAL L., 20II, Gènes et comportements à l'ère post-génomique, Paris, Vrin.

PLAS R., 20 II, «La psychologie cognitive française dans ses relations avec les neurosciences. Histoire, enjeux et conséquences d'une alliance», Revue d'histoire des sciences humaines, 25, p. 125-142.

PROUST J., 1992, «L'interdisciplinarité dans les sciences cognitives», in Entre savoirs. L'interdisciplinarité en acte: enjeux, obstacles, perspectives, E. Portella (dir.), Toulouse, Érès.

RAMUS F., 2005, «De l'origine biologique de la dyslexie», Psychologie \& Education, p. $81-96$.

-, 2011, «Repérer les enfants en difficulté dès la maternelle, une nécessité», Libération, 14 décembre.

RINGARD J-C., À propos de l'enfant "dysphasique" et de l'enfant "dyslexique", Ministère de l'Éducation nationale. 
ROCHEX J-Y., 2000, «La "théorie" du handicap socio-culturel: une explication ethnocentriste qui n'explique rien», Dialogue, 96-97, p. 6-10.

-, 2010, «Les trois "âges" des politiques d'éducation prioritaire: une convergence européenne?», in L'École démocratique. Vers un renoncement politique?, C. Ben Ayed (dir.), Paris, Armand Colin.

- et CRINON J., 20II, La Construction des inégalités scolaires. Au cœur des pratiques et des dispositifs d'enseignement, Rennes, PUR.

ROSE N. et ABI-RACHED J., 2013, The New Brain Sciences and the Management of the Mind, Princeton, Princeton University Press.

TERRAIL J.-P., 2007, «La syllabique est-elle réactionnaire?», in Apprendre à lire. La querelle des méthodes, G. Krick, J. Reichstadt, J.-P. Terrail (dir.), Paris, Gallimard. -, 2013, Entrer dans l'écrit. Tous capables?, Paris, La Dispute.

THÉLOT C., 2004, Pour la réussite de tous les élèves: rapport de la Commission du débat national sur l'avenir de l'École, Paris, La Documentation française.

TIBERGHIEN G., 2002, Dictionnaire des sciences cognitives, Paris, Armand Colin.

TORGENSEN J., 2002, «The Prevention of School Difficulties», Journal of School Psychology, 40-I, p. 7-26.

WOOLLVEN M., 2012, «Expertise réservée, expertise partagée. Les professionnels de la dyslexie en France et aux Royaume-Uni», Carrefours de l'éducation, 37, p. 95-109. 
